\title{
A CONCEPT FOR MODELLING BATCH PLANT OPERATION
}

\author{
J. SIMENSEN ${ }^{*}$ and B.A. FOSS \\ Department of Engineering Cybernetics, The Norwegian University of Science and Technology, \\ N-7034 Trondheim, Norway
}

\begin{abstract}
A model for describing batch plant operations based on three basic components is proposed. The three parts represents different views on the problem. The batch objects follow the batch units moving through the plant. The resource objects document relevant features of each process unit. Finally, the control objects include production plans and recipes. We discuss a rationale for this partitioning and argue that our concept gives a complete view of the process for plant operation purposes. The novel feature of the concept is the integration of essential perspectives to the operation of a pharmaceutical batch plant into one structured information model. This model takes a broader view than just process control and might be used as an agent to integrate and coordinate operation tasks to improve plant efficiency.
\end{abstract}

Keywords: Batch Operation; Modelling; Integration; Pharmaceuticals; Object Orientation.

\section{INTRODUCTION}

Plant operation is a complex task including all the tasks undertaken by the working organization, and the information and control system at the process plant. This includes surveillance and control, production planning, inventory management of the production facilities, as well as minor changes of the production facilities that can be performed by in-house workers. Hence, both the organization, and the information and control system are available resources for running the plant.

In this paper we focus on the structure of the information and control system, in particular we propose an architecture aimed at batch processing plants in the pharmaceutical industries. Process information is essential in the pharmaceutical industries, regulated by the Good Manufacturing Practice (GMP) guidelines provided by i.e. the Federal Food and Drug Administration (FDA) in the U.S., where quality control and documentation is of particular importance. In the extreme a pharmaceutical product without the necessary documentation may be worthless.

In today's competitive business environment there is an increasing focus on and need for efficient process plant operation. A way to achieve this is to improve control at all levels in the control hierarchy. Optimal information utilization is fundamental to efficient process plant operation because information is a most valuable resource for improving control of the manufacturing process. Information on interaction between the various process operation tasks should be considered valuable for control purposes. The rationale for this, is that the process operation tasks interact strongly and should not be treated as individual problems (Pekny et al, 1991). However, all too often the tasks are treated individually, resulting in poor process operation. Plant integration has up to recently mainly been adopted by the manufacturing industry where CIM has become a well known concept. As profit margins are being reduced, however, there is an increasing need for integration in the pharmaceutical industry, too.

Rippin (1993) identifies pioneering work by Cott and Macchietto (1989) integrating the synthesis of operating procedures and scheduling. Other work in the batch area has resulted in an integrated approach to the preliminary design of process, equipment and batch control code, and optimal scheduling (Crooks et al, 1992). Experience shows, however, that there are additionally relevant aspects when operating a batch plant. Dean (1991) identifies process design, production control and monitoring, maintenance, quality assurance, accounting and management as associated tasks.

\footnotetext{
* To whom correspondence should be addressed.

E-mail: jos@itk.unit.no

Fax: $\quad+4773594399$
} 
Process plant operation is made up of various activities and cannot be characterized from a single point of view. Thus, a model framework that is capable of representing the essential perspectives of operation and supporting the integration and coordination of operation tasks in a pharmaceutical batch plant is needed. The need for an information model with a broader view than just process control is also identified by the European Batch Forum (Delhez, 1994). Hence, our challenge is to develop such a concept which can represent these essential perspectives and at the same time is suitable as a basis for an information and control system. Such a basis will also be an agent for tighter control of the manufacturing process at all levels. As multiple process operation views are available though one information and control system, coordination and integration of operation tasks can easily be performed. Our model concept can also serve as a catalyst for improvements in process and operation procedures, as good control is a condition for continuous improvement.

The continuation of this paper is organized as follows: First, our modelling concept is presented on an overall level. Thereafter the different parts of the concept is discussed in more detail. The examples used in this part of the paper are related to the Nycomed Imaging Plants in Norway. Due to limited space we can not give a detailed description of the concept, but we will illustrate the structure and the building blocks of the model concept. Finally, we examine how our framework facilitate the representation of different views on the process.

\section{THE CONCEPT}

Plant operation involves a large number of operational decisions made on different time scales, from long-term production plans to short-term decisions being made by the control system. A single uniform all-knowledge representation technique covering all aspects of a process plant does not exist, as knowledge representation and use cannot be completely separated (Årzén, 1993). Hence, we base our concept on multiple views of the plant derived from the various operation tasks. These perspectives are: 1) The Functional view which pivot processing, product quality and process control. 2) The Quality Assurance view emphasizing traceability, documentation and quality control. 3) The Economical view stresses the utilization of raw materials, production capacity, energy and number of hands. 4) The Technical view focuses physical equipment, technical specifications and maintenance. By examining the various operation tasks we have identified some basic components, and combinations of them, which we believe are capable of representing the various process views. These fundamental building blocks make it possible to establish a concept for modelling batch plant operation. The building blocks to provide this are:

1. Batch objects

2. Resource objects (process and control equipment).

3. Control objects (production plans, recipes, routines).

A batch is a uniquely identified object 'floating' through the process plant according to a recipe. On its 'tour' through the plant, the batch object collects process data from the process control system. When a recipe has been completed, the corresponding batch object contains the complete process documentation. The process plant is viewed as a structure of resources. These resources contain technical specifications, maintenance information and process related information such as process yield based on statistics. Process control loops is also a part of these resources. The control objects controls the execution of batches. A control object can be either general or it can be batch-specific. Examples of the first are control algorithms while examples of the latter are control recipes which are uniquely connected to specific batch-objects. Above we use the term object since every distinct part of the model will be an object in the object-orientation sense of the word (Booch, 1991). Hence, each object contains a data-structure with a well-defined set of operations on it. In addition, access to the data is limited by the encapsulation principle.

\section{BATCH OBJECTS}

On this level, we view batch processing as batch units moving through a structure of resources. This topological abstraction is illustrated by the example process (fig.1) where the batch $\mathrm{Ba} 1$ resides in the resource Reactor 1 .

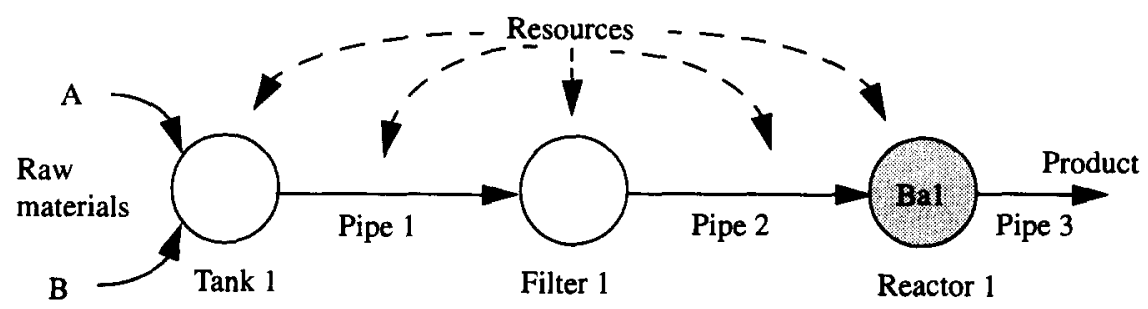

Fig. 1 Process path structure 
One batch is uniquely identified by its batch number. We will use the same batch number to identify the corresponding batch object. On its 'tour' through the plant, the batch object collects process data related to the corresponding batch. When a recipe is completed, the batch object contains the complete process documentation. Together with the physical substance produced, this documentation constitute the pharmaceutical bulk product.

All information on discrete events, like start and stop of process stages, time and results of LAB-analysis, and time, volumes and references (batch-no.) of mixtures are included in the batch objects. Process data from continuous processing is also included. To ensure complete traceability it is necessary to record who performed the manual operations, performed LAB-analysis, initialized automatic operations and so on. Operator signatures for this kind of operations will have to be included in the batch object to complete the batch history. A batch-object is illustrated in fig. 2.

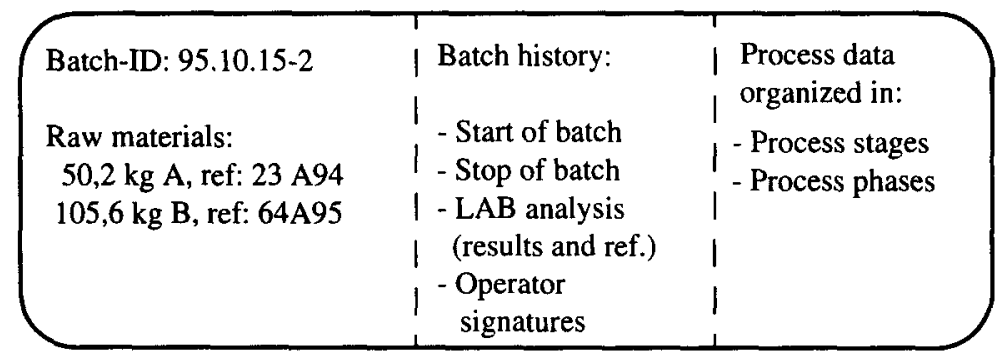

Fig. 2 Batch-object example

\section{RESOURCE OBJECTS}

Our choice of resource objects is based on a topological view of the batch plant. Process equipment like reactors, vessels, filters, containers and pipelines which are used for processing, transporting or storing of batches are looked upon as resources. In a pharmaceutical plant every equipment item is identified by a unique code, which we will use as identifiers for the resource objects. Resources are only used during the processing of a batch. The resource is renewed after cleaning and can then be utilized by a new batch. A resource object contains two main types of information on the equipment item. First, hardware specifications like the manufacturer, time of installation, size and construction materials. Second, operation related information like references to operating procedures, statistical information, e.g. process yield for a reactor, and equipment state. The equipment state can be defined by the set \{ready, occupied, cleaning, maintenance\}. Resource objects with the same attributes can be arranged in a class. Long term production planning can then be based on general resource classes while the assignment of a specific resource to a specific batch can be carried out just before it is needed, to improve the plant operation flexibility.

A resource object can have one or more sub-resources attached to it, related to auxiliary equipment, logs and equipment models. Auxiliary equipment which may need a description is the agitator, the temperature transmitter (TT) and the control valve (V1) shown in fig. 3.

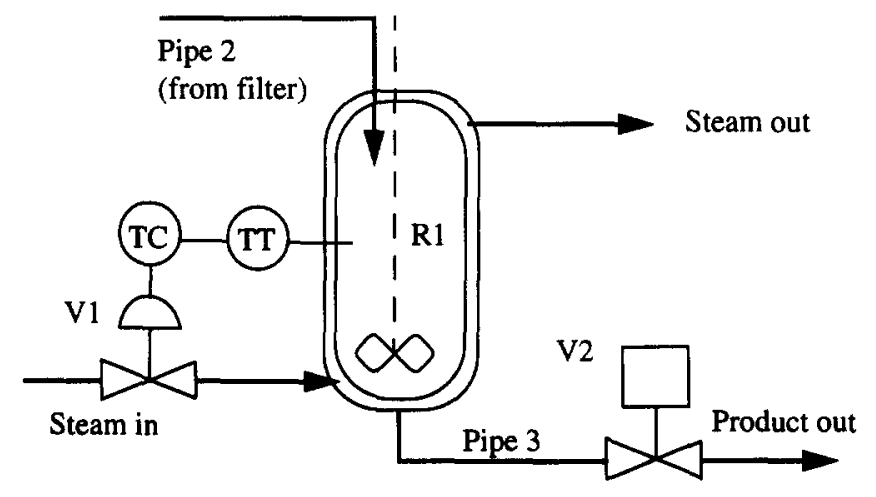

Fig. 3 Example reactor 
Several logs are needed to keep trace of production which is of overall importance in the manufacturing of pharmaceuticals. An example is the maintenance log describing when a piece of equipment has gone through maintenance, what has been done by whom and the planned time for the next maintenance operation. Other kind of logs are the batch log, which keep references of all batches processed by a piece of equipment, and the operation log where operators can put information on process experiences, such as errors in measurements, diagnosis and handling of malfunctions, etc. As we see, logs are important to ensure documentation and traceability on the process equipment side, we will therefore add 'log' as a sub-object to resource objects.

Other possible sub-objects to resources are dynamic models. These can be utilized for control purposes, simulation, optimization, etc. A typical batch situation is characterized by unsteady-state operation (Terwiesch et al, 1994). Thus, a well-suited model is a function of the physical process unit, product, process and time. A convenient scheme is to link the basic unit model to the corresponding resource-object and to complement the model with batch-specific information from the batch-object e.g. product type and batch size.

The resources are organized in a hierarchical structure as illustrated in fig. 4 for the example reactor.

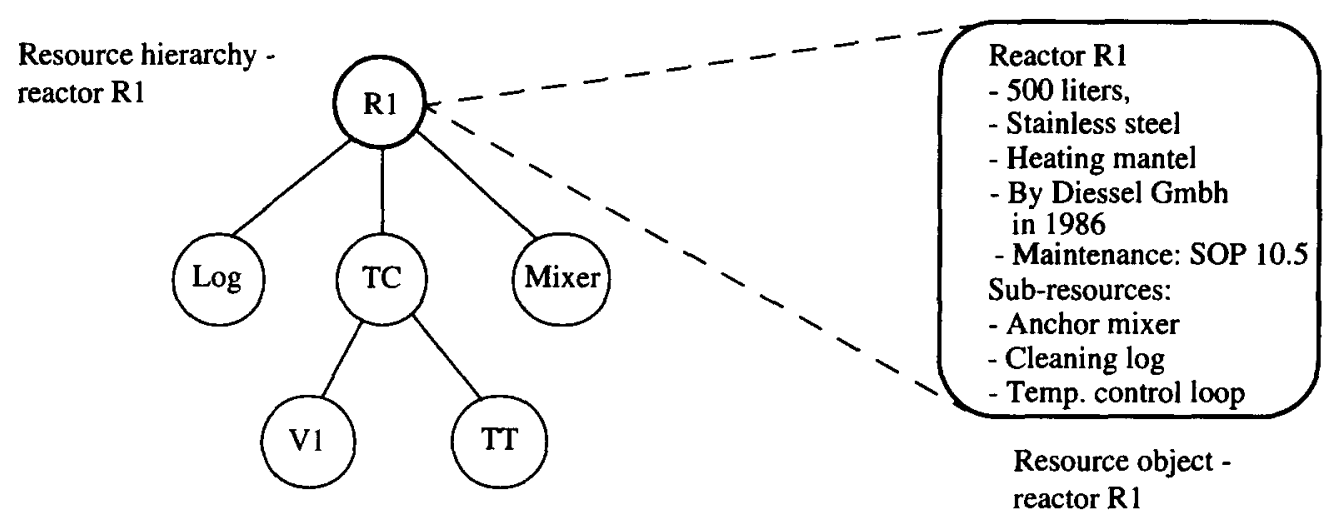

Fig. 4 Resource hierarchy and resource object of example reactor.

\section{CONTROL OBJECTS}

Control-objects are descriptions that controls the execution of batches e.g. production plans, recipes and operation procedures. A control object can be either general or it can be batch-specific. Examples of the first are control algorithms while examples of the latter are control recipes which are uniquely connected to specific batch-objects. The various control-objects corresponds to the three-level control hierarchy presented by Cott and Macchietto (1989) as shown in fig. 5.

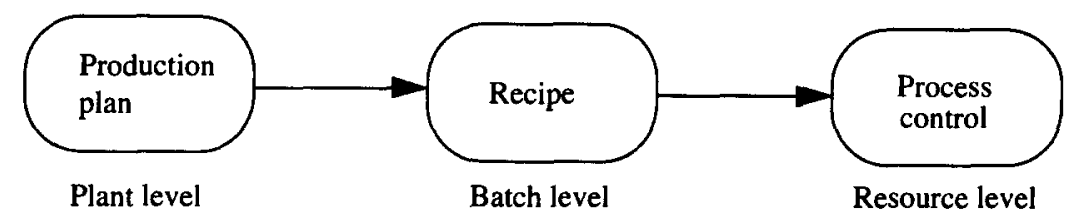

Fig. 5 Process control hierarchy

\section{Production Plan}

Production plans are the results of the production planning activities: that is the allocation of production capacity, production times, labour and energy resources so as to meet market demands for products over an extended period of time into the future, subject to raw, intermediate and final product inventories (Hax, 1978). In the off-line batchscheduling activities plant-wide resources are assigned to the individual batches based on product specific master recipes. These recipes should include estimated needs of processing time for the various resource categories. On-line re-scheduling though, due to inevitable operational disturbances, should be based on batch-specific control recipes and batch-objects to utilize current knowledge of the plant state. 


\section{Recipes}

There are two types of recipes needed to produce a batch (ISA, 1994). First, the master recipe is a unique specification of how to produce a product within a specific process cell. Second, the control recipe starts as a copy of a master recipe and is then modified to a specific batch. These modifications are assignment of a batch number and process equipment, references to raw materials batches, etc. This means that the control recipe contains information necessary to initiate the production of a particular batch. A master recipe has a one-to-one link to a particular product, while a control recipe also has a one-to-one link to a specific batch.

A control recipe controls the transfer of a batch from one resource to the next, assigned by the scheduling, according to some criteria specified in the control recipe. Criteria for the transfer can be determined by 1) experience (LAB-, pilot- or full scale), 2) LAB-tests, 3) on-line measurements or 4) modelbased estimation of process parameters. Similarly, there can be internal transitions between process phases within the same resource. These internal transitions, e.g. from reaction to cooling in a reactor, are very common in batch processing.

\section{Process Control}

Process control at the resource level is the control activity of performing what is usually thought of as "traditionally process control". It encompasses both regulatory and procedural control, as well as the gathering and displaying of data. Procedural control can be diversified into sequential control and operational procedures. Such routines shall, according to GMP, be documented in e.g. Standard Operating Procedures (SOPs). Here we will divide these into three groups. First, there are Process SOPs which describes procedures directly involved in processing, like taking samples for LAB-analysis. Second, Support SOPs describes procedures that are not directly involved in processing, like equipment maintenance. Third, the General SOPs translate quality assurance policy into procedures that serve as general guidelines for operation, e.g. hygiene, general cleaning procedures and safety issues.

The process control code is a system specific translation of the control recipe which can be executed by the process control system. We might say that the process control code is a type of control object, while the control hardware is incorporated into the resource objects. In this way process control represents a link from control object to resource object.

\section{OBJECTS AND OPERATIONAL VIEWS}

In the following we will illustrate how our framework facilitates representation of the various operational views described earlier, as shown in table 1.

Table 1 : Objects and views

\begin{tabular}{l|l|l}
\hline \multicolumn{1}{c|}{ View } & \multicolumn{1}{|c|}{ Operation tasks } & Object type(s) \\
\hline Functional & Processing, process control & $\begin{array}{l}\text { Control and } \\
\text { Resource }\end{array}$ \\
\hline Quality Assurance & Documentation, quality control & $\begin{array}{l}\text { Batch, Control } \\
\text { and Resource }\end{array}$ \\
\hline Economical & $\begin{array}{l}\text { Utilization of materials, capacity, energy, } \\
\text { work force }\end{array}$ & $\begin{array}{l}\text { Control and } \\
\text { Resource }\end{array}$ \\
\hline Technical & Maintenance & Resource \\
\hline
\end{tabular}

Both resource and control objects must be included to represent the functional view, incorporating both process and process control. The first aspect is illustrated by the process path structure in fig. 1, where the process structure is given by the control recipe. The latter aspect is presented in the following simple example: Procedural control of example reactor R1 (resource object) are provided by the control recipe (control object), a batch specific object that also supplies the heating mantel temperature control loop with a setpoint. The temperature control algorithm, though, is a general control object.

As shown in table 1, the quality assurance (QA) view is represented by all three object types. Traceability is met by the batch object. To make up a complete batch documentation, though, the control recipe must be included. As explained earlier, various logs are needed for equipment tracking. Hence, resource objects are also needed to describe the QA-view. The QA-view is illustrated in fig. 6 
The technical view can be represented by resource objects alone. Taking an economical view, the combination of master recipes and the assemble of process equipment is appropriate for scheduling issues.

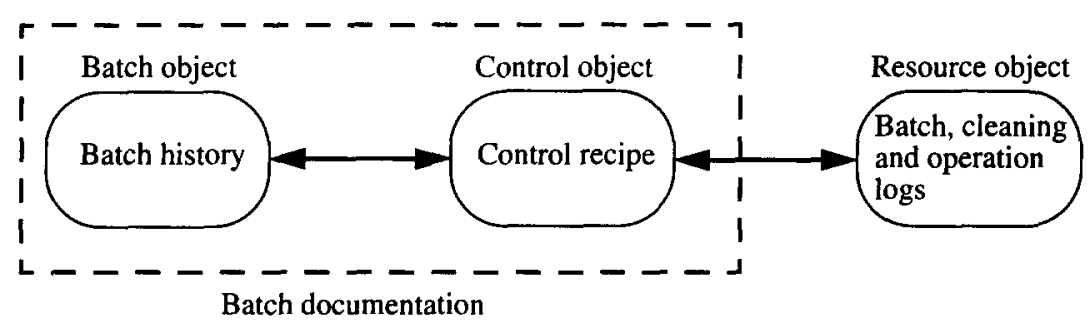

Fig. 6 The quality assurance view

\section{SUMMARY}

We have proposed an abstraction of pharmaceutical batch plant operation consisting of three distinct parts: 1) Resources, 2) Batches and 3) Control objects. Further, we have indicated how this framework can be utilized to model batch plant operation so as to form a basis for an information and control system for batch plant operations. The novel feature of the concept is the integration of essential perspectives of operating a pharmaceutical batch plant into one structured information model. Hence, this model takes a broader view than just process control and should be used as an agent to integrate and coordinate the operation tasks to improve plant efficiency.

\section{ACKNOWLEDGEMENTS}

This work was financially supported by the Research Council of Norway, the Norwegian Federation of Process and Manufacturing Industries (PIL) and the Nycomed Imaging plants in Norway.

\section{REFERENCES}

Booch, G., 1991, Object-Oriented Design with Applications, The Benjamin/Cummings Publ. Comp.: Redwood City, USA.

Cott, B.J. and Macchietto S., 1989, An Integrated Approach to Computer-Aided Operation of Batch Chemical Plants. Comput. Chem. Engng., 13, 1263-1271.

Crooks, C.A., Kuriyan, K. and Macchietto, S., 1992, Integration of Batch Plant Design, Automation, and Operation Software Tools, Comput. Chem. Engng., 16S, S289-S296.

Dean, G., 1991, Meeting the Demands of Batch Control. International Conference on Control '91. Edinburgh, UK, 25-28 March 1991, p. 881-886.

Delhez, J., 1994, European Batch Forum (EBF) - Workgroup 1 Charter and Objectives. 16 November 1994, Draft.

Hax, A.C., 1978, Aggregate production planning. Handbook of Operations Research Models and Applications, Van Nostrand Reinhold, New York.

ISA, 1994, Instrument Society of America, BATCH CONTROL Models and Terminology, March 1994, Draft 10.

Pekny, J., Venkatasubramanian, V. and Reklaitis, G.V., 1991, Prospects for Computer-Aided Process Operations in the Process Industries. Computer-oriented process engineering, Elsevier Science Publishers B.V., Amsterdam.

Rippin, D.W.T., 1993, Batch Process Systems Engineering: A Retrospective and Prospective Review. Comput. Chem. Engng., 17S, S1-S13.

Terwiesch, P. Agarwal, M. and Rippin, D.W.T., 1994, Batch Unit Optimization with Imperfect Modelling: A Survey J. Proc. Cont. 1994, Volume 4, Number 4, 238-258.

Årzén, K.-E., 1993, Using Multi-View Objects for Structuring Plant Databases. Intelligent Systems Engineering, Volume 2, Number 3, Autumn 1993, 183-200. 